\title{
Recuperación dependiente de estados afectivos en una tarea de reconocimiento.
}

\section{Manuel J. Blanco, Fernando del Valle-Inclán y Juan Lamas}

Departamento de Psicologia Fisiológica, Universidad de Santiago.

Las relaciones entre memoria y afecto (en este trabajo utilizamos «afecto», «emoción» y «estado afectivo» como sinónimos) han sido objeto de muchas teorías clásicas, ninguna de ellas satisfactoria (la revisión de Rapaport, 1942, sigue siendo tan actual como entonces). En general, estas teorías sólo consideran la influencia del afecto en la fase de adquisición y no en la de recuperación. El afecto, pues, sólo sería un elemento importante del contexto de adquisición o codificación. Algunos autores han propuesto una clasificación de estas teorías en dos grandes categorías: teorías de la cualidad y teorías de la intensidad. Las primeras tienen como hipótesis central que la cualidad de los afectos (en otras palabras, que sean positivos, negativos o neutros) determina el mayor o menor índice de recuerdo. El ejemplo más típico de este grupo de teorías es la formulación psicoanalítica. Por el contrario, las teorías de la intensidad (ver Dutta y Kanungo, 1975) defienden que el recuer- do depende de la intensidad emocional durante la adquisición. Sin embargo, a nivel metodológico no existen diferencias, pues ambos grupos de teorías emplean el «paradigma experimental de codificación» (Tulving, 1983), manipulan las condiciones (afectivas) de adquisición y mantienen constantes (o no controlan) las de recuperación.

Desde hace algunos años, el interés de los investigadores ha ido hacia las condiciones y proceso de recuperación (Craik, 1981). No niegan la influencia del afecto en la adquisición. Más bien, defienden que existe interacción entre las influencias del afecto en las dos fases (adquisición y recuperación), de forma que la recuperación depende de la congruencia o similitud entre el estado afectivo de adquisición y el de recuperación. El nombre genérico que designa esta interacción es el de «recuperación dependiente de estados afectivos». La mayoría de estos experimentos emplea lo que Tulving (1983)

La información referente a este trabajo debe dirigirse a:

F. del Valle-Inclán, Dpro. Psicología Fisiológica, Facultad de Filosofía y CC de la Educación, Santiago. 
denomina «paradigma experimental de codificación/recuperación». En su forma más simple, este paradigma experimental adopta un diseño factorial $2 \times 2$, donde el primer factor se refiere al estado afectivo de adquisición y el segundo al de recuperación.

Nuestro trabajo se enmarca dentro de esta corriente y estudia la dependencia de estados afectivos en una prueba de reconocimiento. Usualmente, los trabajos sobre la dependencia de estados afectivos emplean tareas de recuerdo libre y los resultados suelen ser favorables a la hipótesis (Bower y Cols., 1978; Bower, 1981; Laird y Cols., 1982, entre otros). Sin embargo, cuando se emplean tareas de reconocimiento (p.e. Macht y Cols., 1977; Bartlett y Santrock, 1979) y de recuerdo con indicios (p.e. Bartlett y Santrock, 1979; Bartlett y Cols., 1982) no suele aparecer el efecto.

A diferencia de estos trabajos, utilizamos un procedimiento hipnótico para inducir estados emocionales, siguiendo a Bower y Cols. (1978), y sustituimos las clásicas listas de palabras por fotografías de caras. El intervalo de retención también fue más largo que lo usual (24 horas). Con estas variaciones encontramos un fuerte efecto de dependencia de estadós afectivos en una prueba de reconocimiento, lo cual obliga a replantearse el papel de la emoción en los procesos de memoria.

\section{EXPERIMENTO}

Empleamos un diseño factorial 2 (estaao afectivo de adquisición) $\times 2$ (estado afectivo de recuperación) con medidas repetidas en los dos factores. Los estados afectivos, inducidos por sugestiones posthipnóticas, fueron alegría y tristeza.

\section{METODO}

\section{Sujetos}

La muestra se compuso de tres mujeres y dos hombres, alumnos de primer curso de psicología, con edades comprendidas entre los 18 y 19 años. Los cinco habían obtenido puntuaciones máximas en la Escala de Grupo de Susceptibilidad Hipnótica de Harvard (HGSHS:A) (Shor y Orne, 1962) y, antes del experimento, ya habían participado en algunas sesiones hipnóticas" individuales. Todos se presentaron voluntarios y ninguno fue remunerado por su participación.

\section{Estímulos}

Empleamos 20 fotografías de caras en blanco y negro tomadas al azar de entre los carnets de alumnos que ya habían acabado la carrera. Las fotografías $(2 \times 3 \mathrm{~cm})$ se presentaron sobre tarjetas $(10 \times 15 \mathrm{~cm})$ de diferentes colores (blanco, negro y verde). El color de la tarjeta se utilizaba como sugestión posthipnótica para inducir cambios en el estado afectivo.

\section{Procedimiento}

Las sesiones se realizaron individualmente y siempre en la misma sala. La iluminación era la usual (no había «luces tenues») durante toda la sesión. En una pared había un espejo bidireccional y en una esquina una cámara de vídeo, que se justificaban como controles experimentales. Uno de nosotros hacía de hipnotizador en todas las sesiones y los otros dos permanecían en una sala contigua observando a través del espejo y controlando la grabación del vídeo. Al final del experimento, proyectamos estas grabaciones a otros investigadores con objeto de controlar las posibles demandas experimentales.

En la fase de adquisición, y después de un breve procedimiento de inducción hipnótica, el sujeto recibía la sugestión posthipnótica de que se sentiría alegre cuando viera unas tarjetas blancas (10 en total) y triste cuando viera unas tarjetas negras (10 también). Tras recibir esta sugestión, se despertaban y se les presentaban las 20 tarjetas. La presentación era en dos bloques atendiendo a la emoción que inducía el color de la tarjetas (alegría y tristeza). Las tarjetas se presentaban una por una y sin limitar el tiempo de exposición. Un dato importante, 
que discutiremos más adelante, fue que los sujetos observaban más tiempo las caras. "alegres» (tarjetas blancas) que las caras «tristes» (tarjetas negras). La tarea del sujeto era observar atentamente las caras y decir si conocía alguna. En ningún momento se le decía que se trataba de una prueba de memoria. Terminada la presentación de tarjetas, se le inducía un trance hipnótico para «anular» las sugestiones anteriores. Finalmente, se le convocaba para una nueva sesión a la misma hora del día siguiente.

La prueba de reconocimiento se hacía 24 horas después de la sesión anterior. Después de un breve procedimiento de inducción hipnótica, el sujeto recibía la sugestión de que se sentiría alegre o triste cuando viera tarjetas de color verde. Despues de comprobar que el sujeto no recordaba esta sugestión, el experimentador presentaba al azar, y de una en una, 40 tarjetas verdes con una fotografía "vieja» (20) o «nueva» (20). El sujeto debía indicar el grado de seguridad de su respuesta (SI o NO) en una escala de cinco puntos.

Una semana después, repetíamos el mismo procedimiento con todos los sujetos, pero induciendo un estado afectivo diferente en la prueba de reconocimiento y utilizando estímulos diferentes. Se le presentaban otras 20 caras (10 sobre tarjetas blancas y 10 sobre tarjetas negras) y, 24 horas después, debían reconocerlas cuando aparecían sobre tarjetas verdes y mezcladas con otras 20 caras «nuevas».

\section{RESULTADOS}

Las variables dependientes fueron tres: porcentaje de éxitos (respuestas «sí» a las caras «viejas»), «área bajo la curva MOC» o $\mathrm{P}(\mathrm{A})$ y criterio de decisión o B. La primera es la medida más usual en este tipo de experimentos. Las otras dos están basadas en el modelo no-paramétrico de la teoría de detección de señales (Green y Swets, 1966). $\mathrm{P}(\mathrm{A})$ o «área bajo la curva MOC» (Memory Operating Characteristic) es una medida de la discriminación entre los ítems «viejos»y «nuevos» independiente del criterio de decisión adoptado por el sujeto en la prueba (ver Banks, 1970; Pastore y Scheirer, 1974).

\section{Porcentaje de éxitos}

En la tabla 1 aparecen los procentajes de éxitos en cada condición y los resultados de un experimento similar de Gellerman y Bower (en Bower y Cohen, 1982). Estos autores no encontraron un efecto de dependencia de estados afectivos con esta medida. Nuestro análisis estadístico (ANOVA con medidas repetidas) mostró una interacción significativa entre los dos estados afectivos (adquisición y recuperación) $(\mathrm{F}(1,4)=$ 10.29 ; $\mathrm{P}<.03$ ). El porcentaje más alto correspondió a la condición tristeza-tristeza (aprendieron y reconocieron las caras con tristeza) y no había diferencias entre las otras condiciones.

\begin{tabular}{|c|c|c|c|c|c|c|c|c|}
\hline \multirow{3}{*}{ 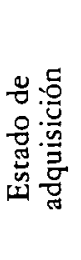 } & \multicolumn{4}{|c|}{$\begin{array}{l}\text { Estado de } \\
\text { reconocimiento }\end{array}$} & \multicolumn{4}{|c|}{$\begin{array}{l}\text { Estado de } \\
\text { reconocimiento }\end{array}$} \\
\hline & Alegría & $\begin{array}{c}\text { Alegría } \\
52\end{array}$ & $\begin{array}{c}\text { Tristeza } \\
56\end{array}$ & $\begin{array}{l}X \\
54\end{array}$ & Alegría & $\begin{array}{c}\text { Alegría } \\
64\end{array}$ & $\begin{array}{c}\text { Tristeza } \\
65\end{array}$ & $\underset{64.5}{X}$ \\
\hline & $\begin{array}{r}\text { Tristeza } \\
\mathrm{X}\end{array}$ & $\begin{array}{l}56 \\
54\end{array}$ & $\begin{array}{l}72 \\
64\end{array}$ & 64 & $\begin{array}{r}\text { Tristeza } \\
\mathrm{X}\end{array}$ & $\begin{array}{l}59 \\
61.5\end{array}$ & $\begin{array}{l}57 \\
61\end{array}$ & 58 \\
\hline
\end{tabular}

b)

TABLA 1. Porcentaje de reconocimientos correctos (éxitos) de caras para las distintas condiciones de adquisición y reconocimiento.

a) Datos de nuestro experimento $(n=5)$.

b) Datos del experimento de Gellerman y Bower. 
Estos datos sólo confirman un efecto de dependencia en la condición de tristeza, pero no en la de alegría. Cuando los sujetos estaban tristes reconocían mejor las caras «tristes» que «alegres», pero en alegría las reconocian igual.

\section{B, Criterio de decisión}

Como medida del criterio de decisión o inclinación de respuesta empleamos B (ver $\mathrm{McNicol}, 1972$ ). Los sujetos no utilizaron todas las categorías de la escala de confianza. Por este motivo, agrupamos las respuestas en tres categorías: 1 (confianza alta), 2 y 3 (confianza media) y 4 y 5 (confianza baja). En la tabla 2 aparecen estos valores B calculados a partir de estas tres categorías (varían entre 1 y $\mathrm{N}-1$, siendo $\mathrm{N}$ el número de categorías de respuesta). En el ANOVA $2 \times 2$ apareció significativa la interacción entre los dos estados afectivos $(F(1,4)=12.04 ; p<.02)$, aunque ninguno de los dos tuvo efectos por separado. Según esto, el tipo de cara («alegre» o "triste») y el estado afectivo de reconocimiento influyen conjuntamente sobre el criterio de decisión adoptado por el sujeto durante la prueba. Los sujetos en tristeza emplean un criterio de decisión más cauto o estricto cuando reconocen caras «tristes», pero en alegría emplean un criterio similar para los dos tipos de caras.

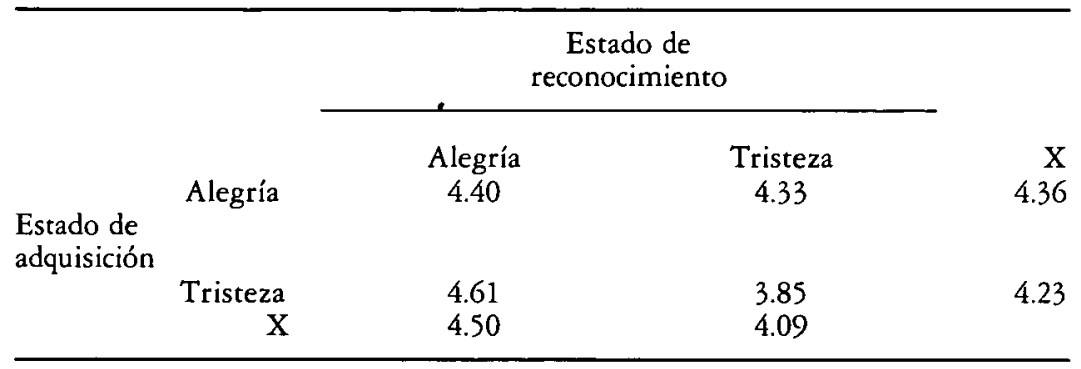

TABLA 2. Valores B (criterio de decisión) del reconocimiento de caras en las distintas condiciones de adquisición y recuperación. Los valores bajos señalan un criterio de decisión estricto.

$\mathbf{P}(\mathrm{A})$, «área bajo la curva $\mathrm{MOC}$ »

El análisis anterior mostró que los estados afectivos de adquisición y recuperación varían el criterio de decisión $B$. $P(A)$ es una medida de discriminación entre los ítems «viejos» y. «nuevos» que, a diferencia del porcentaje de éxitos, es independiente de este criterio. En la tabla 3 aparecen los valores $P(A)$ (varian entre 0.5 y 1.00 ).

\begin{tabular}{|c|c|c|c|c|}
\hline \multirow{3}{*}{$\begin{array}{l}\text { Estado de } \\
\text { adquisición }\end{array}$} & & \multicolumn{2}{|c|}{$\begin{array}{l}\text { Estado de } \\
\text { reconocimiento }\end{array}$} & \multirow[b]{2}{*}{$\begin{array}{r}\mathrm{X} \\
77\end{array}$} \\
\hline & Alegría & Alegria & Tristeza & \\
\hline & Tristeza & .76 & .85 & .80 \\
\hline & $X$ & .77 & .80 & \\
\hline
\end{tabular}

TABLA 3. Valores $P(\dot{A})$, kárea bajo la curva $\mathrm{MOC}_{*}$, del reconocimiento de caras en las distintas condiciones de adquisición y reconocimiento. Estos valores fueron transformados a $2 \operatorname{arcsen} P(A)$ antes del análisis de varianza (ANOVA). 


\section{Estudios}

Los datos indican un claro efecto de dependencia: los valores más altos corresponden a las condiciones en las que los estados afectivos de adquisición y reconocimiento eran iguales (.79 para la condición alegrealegre y .85 para la condición triste-triste). Antes de proceder al ANOVA de medidas repetidas, transformamos estos valores en 2arc sen $P(A)$, siguiendo las indicaciones de McNicol (1972) (ver también Swets y Pickett, 1982). Los resultados de este análisis no mostraron efectos significativos del estado de adquisición $(\mathrm{F}(1,4)=.62 ; \mathrm{ns}) \mathrm{ni}$ del de reconocimiento $(F(1,4)=.02 ; n s)$. Sin embargo, apareció significativa la interacción entre los dos $(F(1,4)=7.75 ; \mathrm{p}<.04)$. Las caras «alegres» se reconocieron mejor en la condición de alegría y las «tristes» en la de tristeza. Este resultado confirma, pues, un efecto de dependencia de estados afectivos.

\section{DISCUSION}

Los resultados indican un efecto de dependencia de estados afectivos en una tarea de reconocimiento de caras. El dato es importante pues, como ya señalamos, estudios anteriores sugieren que este efecto sólo ocurre con tareas de recuerdo libre. Destacamos tres razones para este desacuerdo: (1) empleamos dos listas de estímulos (caras) en la fase de adquisición, mientras que la mayoría de los experimentos emplean una única lista; (2) el intervalo de retención (24 horas) fue más largo que el de otros estudios (generalmente, entre 10 y 30 minutos); (3) el análisis de datos basado en la teoría de detección de señales proporciona un índice más preciso y fiable (área bajo la curva MOC) de la discriminación entre ítems «viejos»y «nuevos» que el porcentaje de éxitos, que ha sido la medida más empleada.

Nuestro diseño es muy similar al de los estudios sobre interferencia. Los sujetos aprendían dos bloques de estímulos en la misma sesión experimental y en diferentes estados afectivos. Bower y Cols. (1978) no encontraron efectos de dependencia con diseños de una única lista de ítems, pero sí con diseños de interferencia (los sujetos aprenden dos listas y deben recordar una de ellas). Pudiera ser que el estado afectivo permita discriminar mejor una lista de ítems cuando se aprenden varias en distintos estados.

Es posible que la efectividad del estado afectivo como indicio de recuperación también aumente con el intervalo de retención. De hecho, algunos trabajos clásicos parecen indicar que los efectos de experiencias emocionales en la memoria perduran durante mucho tiempo (ver Rapaport, 1942). Si esta inferencia es correcta, debemos esperar que el efecto de dependencia sea mayor con intervalos largos que con intervalos cortos. Esta variable precisa un estudio sistemático. En estos momentos sólo podemos comparar nuestros resultados con los de otros estudios.

El tercer factor que ha contribuido a nuestros resultados es el análisis de datos basado en la teoría de detección de señales. El porcentaje de éxitos, que es la medida usual en este tipo de experimentos, no parece una medida adecuada de reconocimiento (ver Swets y Pickett, 1982), puesto que varía con el criterio de decisión adoptado por el sujeto durante la prueba. En uno de nuestros análisis, encontramos que este criterio variaba en función del estado afectivo. Precisamente, el aspecto más relevante de la aplicación de la teoría de detección de señales al estudio del reconocimiento es que permite controlar o especificar el criterio de decisión. En este experimento encontramos un claro efecto de dependencia con el «área bajo la curva $\mathrm{MOC}, \mathrm{P}(\mathrm{A})$, como variable dependiente. 
Estudiamos los efectos de los estados afectivos de adquisición y recuperación en una tarea de reconocimiento. Los estados afectivos se indujeron por sugestiones posthipnóticas $y$ los estimulos fueron fotografias de caras en blanco y negro. Empleamos un diseño factorial 2 (estado de adquisición) $\times 2$ (estado de recuperación) con me. didas repetidas. El intervalo entre las fases de adquisición y recuperación fue de 24 boras. El análisis de datos, basado en el modelo no-paramétrico de la teoria de detección de señales, confirma el efecto de dependencia de estados afectivos en una prueba de reconocimiento. Los resultados se discuten en relación a trabajos anteriores que no babian encontrado este efecto en pruebas similares.

\section{Abstract}

The present experiment studies the influencies of adquisition and retrieval mood on a recognition task. Affective states were induced by posthypnotic suggestions and stimuli were fotographs of faces. We use a 2 (ad. quisition state) $\times 2$ (retrieval state) factorial design with repeated measures. The interval between adquisition and retrieval was 24 hours. Data was treated following the non-parametric model of signal detection theory. These data confirm a effect of affective state dependence in a recognition task. The results are discussed with previous experimental work where no significant effects bave been reported.

\section{Referencias}

BANKS, W.P. Signal detection theroy and human memory. Psychological Bulletin, 1970, 74, 81-99.

BARTLETT, J.C., BuRleson, G. y SANTROCK, J.W. Emotional mood and memory in young children. Journal of Experimental Child Psychology, 1982, 34, 59-76.

BARTLETT, J.C. y SANTROCK, J.W. Affect-dependent episodic memory in young children. Child Development, $1979,50,513-518$.

Bower, G.: Mood and memory. American Psychologist, 1981, 36, 129-148.

BOwER, G. y CoHEN, P.R.: Emotional influences on memory and thinking: data and theory. En S. Fiske y M. Clark (Eds.), Affect and Cognition. Hillsdale, N.J.: Erlbaum. 1982.

Bower, G., MonTeiro, K.P. y Gilligan, S.G.: Emotional mood as a context for learning and recall. Journal of Verbal Learning and Verbal Behavior, 1978, 17, 573-585.

CRAIK, F.I.M.: Encoding and retrieval effects in human memory: a partial review. En J. Long y A. Baddeley (Eds.), Attention and Performance (Vol. IX). Hillsdale, N.J.: Erlbaum, 1981.

DutTA, S. y KanUngo, R.N.: Affect and memory: a reformulation. Oxford: Pergamon Press, 1975.

GreEN, D.M. y SWETS, J.A.: Signal detection theory and psychophysics. Nueva York: Wiley, 1966.

LAIRD, J.D., WAGENER, J.J., HAlal, M. y SzEGDA, M.: Remembering what you feel: effects of emotion on memory. Journal of Personality and Social Psychology, 1982, 42, 646-657.

MaCht, M.L., SPEAR, N.E. y LEvis, D.J.: State-dependent retention in humans induced by alterations in affective state. Bulletin of the Psychonomic Society, 1977, 10, 415-418.

McNicol, D.: A primer of signal detection theory. Londres: G. Allen \& Unwin Ltd, 1972.

PASTORE, R.E. y SCHEIRER, C.J.: Signal detection theory: considerations for general applications. Psychological Bulletin, 1974, 81, 945-958.

RAPAPORT, D.: Emotions and memory. Nueva York: International Univ. Press, 1942.

Shor, R.E. y ORNE, E.C.: Harvard Group Scale of Hypnotic Susceptibility, Form A. Palo Alto, California: Consulting Psychol. Press, 1962.

SweTs, J.A. y PICKETT, R.M.: Evaluation of diagnostic systems. Nueva York: Academic Press, 1982.

Tulving, E.: Elements of episodic memory. Oxford: Univ. Press, 1983. 